International Journal of Artificial Intelligence and Applications (IJAIA), Vol.8, No.2, March 2017

\title{
SHORT-TERM FORECASTING OF ELECTRICITY CONSUMPTION IN PALESTINE USING ARTIFICIAL NEURAL NETWORKS
}

\author{
Shorouq Salahat ${ }^{1}$ and Mohammad Awad ${ }^{2}$ \\ ${ }^{1}$ Master Students in Faculty of Engineering and Information Technology, Computer \\ Science Department, Arab American University-Jenin, Palestine \\ ${ }^{2}$ Faculty of Engineering and Information Technology Computer Engineering Department, \\ Arab American University-Jenin, Palestine
}

\begin{abstract}
Nowadays, planning the process of electricity consumption demand is one of the keys success factors for the development of countries. Due to the importance of electricity, countries have greatly paid attention to the prediction of electricity consumption. Electricity consumption prediction is a major problem for the power sector; an efficient prediction will help electrical companies to take the right decisions and to optimize their supply strategies for their work. In this paper, we proposed a model that is used to predict the future electricity consumption depending on the previous consumption. This model provides companies and authorities to know the future information about the electricity consumption, so they can organize their distribution and make suitable plans to maintain the stability in the delivery and distribution of electricity. We aim to create a model that will be able to study the previous electricity consumption patterns and use this data to predict the future electricity consumption. The system analyzes the collected data of electricity consumption of the previous years, then byusing the mean value for each day and the use of Multilayer Feed-Forward with Backpropagation Neural Networks (MFFNNBP) as a tool to predict the future electricity consumption in Palestine. The data used in this paper depends on data collection of months and years. Finally, this proposed model conducts a systematic process with the aim of determining the future electricity consumption in Palestine. The proposed application and the result in this paper are developed in order to contribute to the improvement of the current energy planning tools in Palestine. The experimental results show that the model performs good results of prediction, with low Mean Square Error (MSE).
\end{abstract}

\section{KEYWORDS}

Artificial Neural Networks, Time Series Prediction, Electricity Consumption.

\section{INTRODUCTION}

Today, demand planning for electricity consumption is a key success factor for the development of any country [1]. Electricity power is one of the most important powers in the today's modern life and it is effective on the welfare level and also the quality and efficiency of job and production [2]. Electricity constitutes one of the main energy sources for our civilization. Its use covers a very large range of activities ranging from purely industrial uses to domestic consumption of families $[15,17]$. The growing and the globally competitive economy are not conceived without the stability of the electricity connection with high-quality, competitively priced [19]. The dependence has turned electrical energy into a strategic input that has intense effects, not only economic but also social. So we can say that we live in a world that works with electricity. We must outline the electricity consumption in the future. Due to the importance of electricity power, governments and organizations, related to it in the developed and developing countries, have greatly paid attention to the prediction of electricity consumption. Because of several years of Israeli occupation of Palestine, Palestine's economy is suffering many of underdevelopment in many sectors; the most major one is energy sector that is very important in

DOI : 10.5121/ijaia.2017.8202 
International Journal of Artificial Intelligence and Applications (IJAIA), Vol.8, No.2, March 2017

any country [3]. In Palestine there are no energy supplying resources, all energy resources are managed by sectors of Israeli occupation that makes energy price very high also the occupation to controlling the amount of energy that will be supplied[4]. Because of this, the Palestinian electrical distributing company start thinking about the necessity of forecasting the electrical consumption to save the ability of supplying the customer as much possible. In order to manage the electricity as much as consumptions, it is necessary to forecast the usage of the future electrical power in the distribution network. The pattern of electrical power usage depends on many different parameters such as the weekdays, seasons, weather conditions etc. [5]. Forecasting can help to determine where saving can be made; the advantage of good forecasting is to get good future predictions using history of data. There are two types of load forecasting: short load forecasting usually a month to a year, and long load forecasting usually in the long history of data [6].

Today, researchers try to forecast the electrical power using different forecasting methods, some of most usage method are autoregressive integrated moving average, multiple linear regression, time series method, and artificial neural network [7]. Also, is tried to find out the pattern of electrical power usage with the dataset which is prepared by real data. Many of proposed models we find in energy load forecasting, in [5] different forecasting methods were utilized to formulate prediction models of the electricity demand in Thailand are autoregressive integrated moving average (ARIMA) [7,16], artificial neural network (ANN) and multiple linear regression (MLR) then compared the performance of these three approaches the results showed that the ANN model has better mean absolute percentage error (MAPE) than ARIMA and MLR model respectively $[7,18]$.

In paper [8] the authors forecast the electrical power according to weather data by using the artificial neural network in Bushehr. The result of this research shows the high efficiency of the neural network (MLP) in estimating the electrical power load. In [1] they proposed a Narx neural network to predict Iran electricity consumption, logarithmical Pre-processing over the input data is used to improve the performance. Also, ARIMA model and Perceptron neural network were used to compare with narx neural network, results showed better electricity consumption using the proposed model. In[9] two techniques for modeling electricity consumption of the Jordanian industrial sector were presented, multivariate linear regression and neuro-fuzzy models. Different variables us as input such as the number of establishments, the number of employees, electricity tariff, prevailing fuel prices, The results showed that the neuro-fuzzy model performs better for prediction of electricity consumption than the multivariate linear regression model both model, a comparison based on the square root average squared error of data. In [10] they compare ANNs approach with ARIMA model on real data for electricity consumption in Gaza Strip, the comparison of performance between the two proposed models reveals that ANNs outperform most forecasting than the ARIMA model. In [15] the authors used artificial neural networks (ANN) to predict Greek long-term energy consumption. They were testing several possible architectures of MLPNN in order to select the one with the best generalizing ability.

In this paper, we proposed an artificial neural networks model which is used to find the best fitting to predict the future electricity consumption in Palestine depending on the previous values of the last four years. The data set is collected from the Nablus city from (Northern Electrical Distribution Company (NEDCO). The model uses Multilayer Feed-Forward with Back propagation Neural Networks (MFFNNBP) as the prediction tool of the future values of the electricity consumption [14]. The real output data will be used as the target of the input which has the input values from 0-30 for month prediction and 1-365 for year prediction. The MFFNNBP start predicts the current output comparing it with the target output to find the mean square error and then it increased the number of neurons by one neuron even get the maximum number of iteration to the threshold value. In section 2 we will show the ANN architecture and the 
International Journal of Artificial Intelligence and Applications (IJAIA), Vol.8, No.2, March 2017

MFFNNBP architecture, section 3 shows our methodology and data pre-processing, the result are discussed in section 4, and finally, theconclusion of the paper will be presented.

\section{Artificial Neural Networks (ANN)}

The neural network is designed to work like human brain neurons cell to solve nonlinear mathematical process [21]. The NN have the ability to learn patterns from input data also can handle large and complex system with many parameters [10]. ANNs are a powerful tool for the approximation of linear functions, Its use is especially useful in the modeling complex functions [11]. The application of this type of techniques in the field of time series prediction has provided interesting results. In general, there are two types of techniques used for Prediction: those based on the classic series analysis or econometric models [22], and those based on the field of artificial intelligence [12]. Neural networks were born from the study of brain functionality, the NNs parallelism has motivated many researchers to propose a new connection models and new learning methods based on the modeling of the brain. The objective is to achieve a generalization and robustness similar to that of the brain. An artificial neural network is a parallel processing structure of distributed information [10], as shown in the following figure:

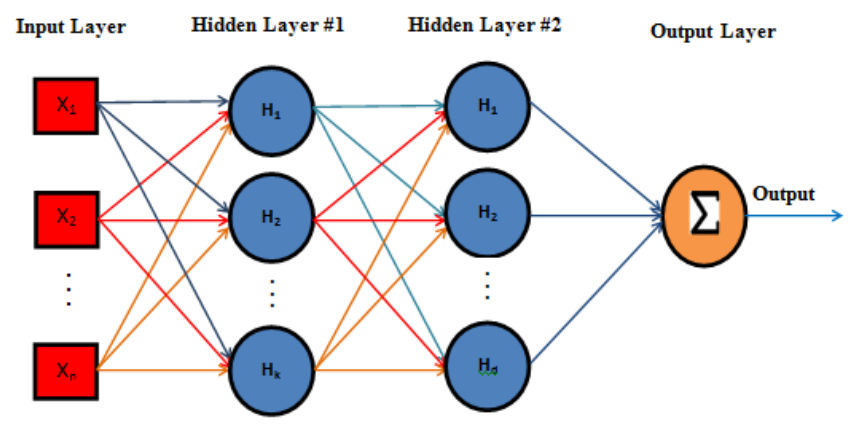

Figure1:MLP Neural Network Architecture.

Artificial neural networks are adaptive structures of information processing, where processing takes place through the interconnection of very simple elements that are called neurons. During the learning phase, the network is exposed to the information environment so you can adapt your weights (parameters that form the transfer functions of their process) and also its structure [10]. During the testing process, the network weights are kept fixed and the network is limited to, the input information that is provided to you. Artificial neural networks owe their processing power to information to its distributed and parallel structure and to its ability of learning and generalization [10]. This makes the networks neurons are able to solve certain types of very complexes that had not been solved until now. The consumption of electricity depends on many variables through nonlinear and very complex that the use of neural networks for their prediction is very adequate. The prediction of the electricity consumption is an element of great Importance for the strategy of all market participants. In Palestine, there hasn't been any attempt to make the electricity consumption.

Basically, NN architecture consists of three layers, input layers, hidden layers, and output layers as shown in figure 1 . The input layers represented by $X(n)$ where $\mathrm{n}$ the number of input data, each of these inputs connected to hidden layer by weights represented by $W$, and the output represented by $Y$. The first step is the initialization of the weights normally random then, we can calculate with the output function of the neural network which is represented as follows: this process is called the forward pass: 


$$
Y_{i}=f\left(\sum_{i}^{k}\left(W_{i j} X_{i j}\right)\right.
$$

Where $k$ is the number of neurons, and $f$ is the activation function are used in the network. To determine the error each NN output $\left(Y_{i}\right)$ will be compared with an observed value $\left(Y_{o b}\right)$ using following equation:

$$
E=Y_{i}-Y_{o b}
$$

Actually, this equation used as criteria to stop the $\mathrm{NN}$ in agood performance as possible as, in this paper, we use the mean square error (MSE) as criteria condition which equation is:

$$
M S E=\frac{1}{n} \sum_{i=1}^{n}\left(Y_{i}-Y_{o b}\right)^{2}
$$

The Backwards Pass start from the goal with back propagation is to update each of the weights in the neural network so that they cause the actual output to be closer the goal output, by minimizing the error for each output neuron and the network as a whole, this process calculated as in the following expression:

$$
\Delta w_{i+1}=\alpha . M S E . X_{i}
$$

The training process will continue until the MSE reach condition criteria value, and update the weights in each iteration using MSE by equation4.

$$
W_{\text {new }}=W_{\text {old }}+\alpha * M S E * X_{i}
$$

Where $\alpha$ is learning rate between 0 and 1 . In each iteration we checked the stop condition if it occurred or not.

The most popular NN type used in load forecasting is multilayer perceptron (MLP) with back propagation. The MLPNN composed of three layers: an input layer, a hidden layer, and output layer. With regard to the input layer, we will locate the number of days, 30 for the month prediction and 365 days for year prediction. The output layer will only have one unit, which will indicate the value of the electrical consumption time associated with each input data presented on the neural network. The hidden layer will have a variable number of neurons. Determination of the optimal number of hidden layer neurons has led to through increased process in which we start with one neuron increase one in each execution, stop when the model get an admissible error.

\section{Proposed Modelmethodology}

The electricity consumption series depends on particular characteristics such as, demand series with strong patterns annual, monthly, weekly, daily Time, electricity consumption varies according to the season of the year, days of the week and time of day. Another characteristic is the highest consumptions occur during the days at peak hours such as 18:00 pm and 00:00 pm [8]. Its evolution in the short, medium and long term is influenced by extreme weather events and factors such as economic growth, energy or population growth. The study of the electricity consumption in time produces a time series problem. Time series prediction takes a set of current data that is used to predict future data. The main goal of time series is to build a model to deduce future unknown data from current data with minimizing the error function between input and output. Establishing a mapping between inputs and outputs is the first step for time series, the mapping is linear and nonlinear. 
Developing a model to predict the relationship as accurately and precisely as possible. The values of future electricity consumption require the knowledge of the previous electricity consumption, these values are used as input to the model, $x_{t}=F\left(x_{t}, \ldots, x_{t-t w}\right)+\varepsilon_{\mathrm{t}}$, where $x_{\mathrm{t}}$ is the forecasting forward steps with respect to time $t, F$ is the modeling function between the previous and future values, $\varepsilon_{\mathrm{t}}$ is the modeling error. An efficient method of predicting with the use of neural networks, Multilayer feed- forward with back propagation neural networks (MFFNNBP) is an MLPNN that passes the inputs and the weights from one layer to the next one through the feed forward process and then it performs the weights update to be back-propagated to the previous layers in order to recalculate the weights $[11,14]$ MFFNNBP is built withinput layer, one output layer, and many hidden layers. The number of neurons in input layer, output layer is determined by the input variables, output variables respectively. But the neurons in hidden layer are determined by the complexity of the problem we solved. In our work we are using neural network fitting tool in Matlab to determined our result, The neural network fitting tool (nftool) leads us to solve data fitting problem with the two-layer neural network, this network is feedforward network type using Leven berg Marquardt algorithm in training [13].

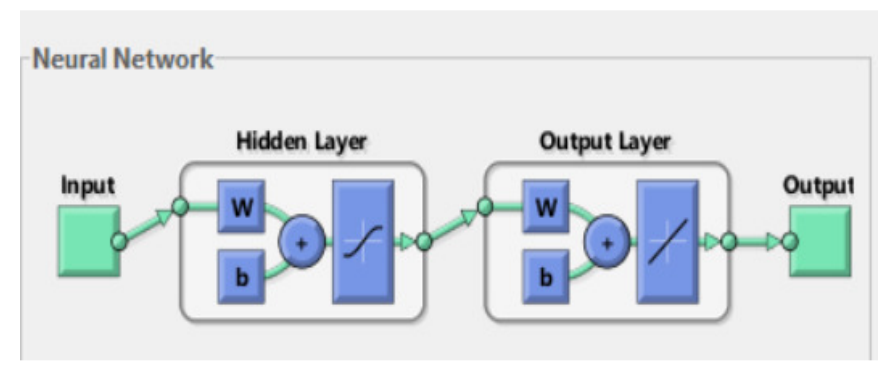

Figure 2:Neural network fitting architecture

In MLPNN, the output of a layer will be an input for the next layer passing from the input layer to the output layer; the equations used for this procedure are illustrated as follows:

$$
\text { output }=f^{2}\left(\sum_{j=1}^{n} \text { out }_{1} \cdot w_{j k}\right)
$$

Where the output of the first hidden layer out , which is calculated using the following expression:

$$
o u t_{1}=f^{1}\left(\sum_{j=1}^{n} i n_{i} \cdot w_{i j}\right)
$$

Where $f^{l}$ and $f^{2}$ are the activation functions for output layer and hidden layer, which is calculated as in the following expressions:

$$
\begin{gathered}
f^{1}=\frac{1}{1+e^{-x}} \\
f^{2}=x
\end{gathered}
$$


Where, $x=$ input vector. We have data set of Nablus city electricity consumption from 2010 to 2014 from NEDCO. Using the average value of electrical consumption from 2011 to 2014 in Nablus city to predict the next year's electrical consumption amount. Before using the data we had done some preprocessing to have better rustle. This processing will be discussed in the next section. Then we apply the data to neural network fitting tool, the input in $\mathrm{NN}$ was $\mathrm{X}$ vector that presents day number from $[1,365]$, and the output is electrical consumption predicted. Also in this paper, we focus in the higher consumption of a month in summer as well as in winter season which are January and July respectively. For each month, we calculated the average for previous 4 years to predict the consumption in the same month next year. The proposed model passes through mean steps and Figure 2 illustrates the proposed MFFNNBP model.

Pre-processing data help networks to have better performance and faster learning. The input variables are pre-processed before being used to train the network. In our work the data have the large scale to pre-processing it, we use two types of algorithms, and the first one is smoothing the data (data value averaging). The second was used to normalize the data (min-max normalization) [15]. In the method data value averaging, data set can be enhanced by taking the average of the data, to smoothing the data we replace each point with the average of the neighboring points as in equation 6

$$
Y_{2}(i+k-1)=\left(Y_{1}(i)+Y_{1}(i+1)+Y_{1}(i+2)+\cdots+Y_{1}(i+k-1)\right) / k
$$

Where $Y_{2}$ is the new data, $Y_{1}$ is the original data, $\mathrm{k}$ is, the number of neighboring points, and $\mathrm{i}$ from 1 to $\mathrm{N}$ where in number of input data.Another method of preprocessing is Min-Max Normalization. Min max normalization is used to rescale the data linearly. It transform the input data scale to, a new scale in [a,b], but generally used in $[0,1]$ scale , the equation(7) shows it.

$$
Y_{\text {new }}=\left(\frac{y_{\text {old }}-\min }{\max -\min }\right)(a-b)+b
$$

Where $Y_{n e w}$ are the normalized data, $y_{\text {old }}$ the original data, and min; max are the minimum and maximum value in the data. Figure 3 show the data before pre-processing, figure 4 show the smoothing on data and figure 5 shows the normalization.

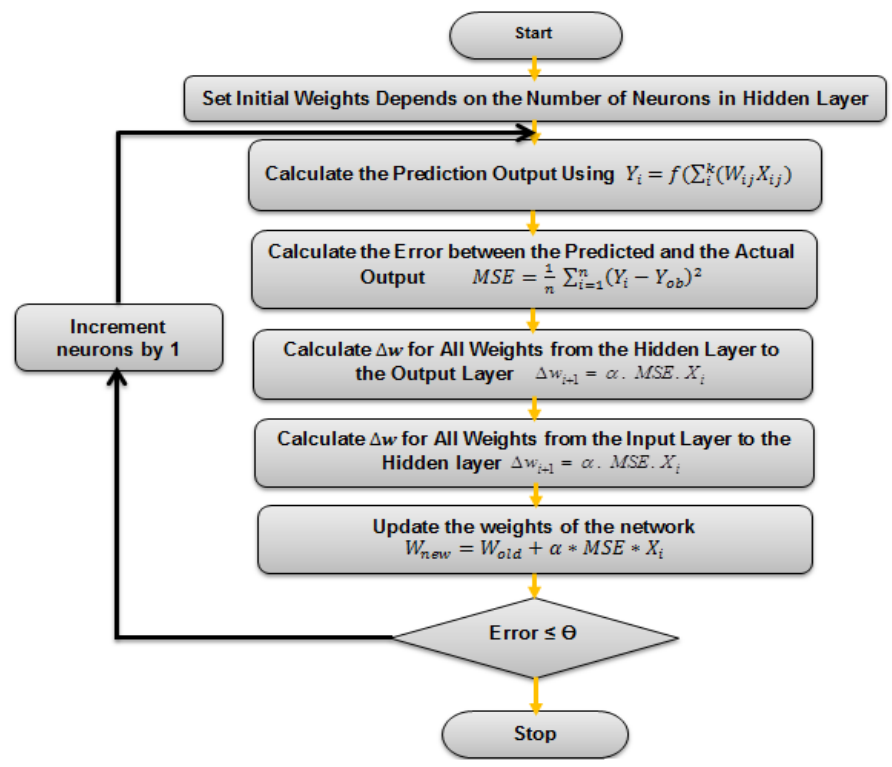

Figure. 2. Process steps of the proposed model (MFFNNBP) 
The process of training the MFFNNBP depends on the backpropagation process, which calculates the gradient decent error between the target output and the predicted output considering the new weights each time.The Levenberg-Marquardt method involves an iterative improvement of values in order to reduce the sum of the squares of the errors. The Levenberg-Marquardt use gradient descent method and the Gauss-Newton method to solve problems [20]. In the gradient descent method, the sum of the squared errors is reduced by updating the values in the direction of the greatest reduction of the least squares objective, and the Gauss-Newton method reduces the sum of the square errors to finding the minimum of the quadratic [13]. Levenberg-Marquardt algorithm updates value between the gradient descent update and the Gauss-Newton update using equation 6.

$$
\left[X^{T} Z X+\lambda I\right] h_{l m}=X^{T} Z(y-\hat{y})
$$

Where $X$ is the Jacobian matrix, $Z$ is weighting matrix, parameter $\lambda$ is initialized to be large, small values of $\lambda$ result in a Gauss-Newton update and large values of $\lambda$ result in a gradient descent update, $\lambda$ is increased if the result in a worse approximation and decreased if the solution approach.

\section{RESUlt AND DisCUSSION}

To execute a real data on the basic architecture of the MFFNNBP model, we collected data from the Northern Electrical Distribution Company (NEDCO) for the previous 4 years for the electricity consumption on Nablus City. We constructed a basic MLPNN model that is sufficiently completed to obtain reliable prediction results. The model allows add several hidden neurons but has no recurrences that greatly increase the complexity of the model, to determine in some way the goodness of the prediction. This determination will carry out the prediction error. In this paper, we use the mean square error as presented in Eq. 3. Before introducing the necessary data to the model it is important to perform the pre-processing step as in Eqs.10 and 11.

The model is simulated in MATLAB R2012b under Windows 8 with processor i7. In this section, the data set is 365 sample of one element (time) in year forecasting and a data set of 31 sample of one element(time) in a month forecasting, in this paper, we divide the input data to $70 \%$ of the input data used as a training set, $15 \%$ used for validation and finally the other $15 \%$ for testing. We have two part in our work, yearly forecasting, and monthly forecasting, the result discussion well be in the next subsection below.

\subsection{YEARLY FORECASTING EXPERIMENT}

For year forecasting, we used data average for the last four years from (2011-2014) which is shown in figure 3, applying the MLPNN model on preprocessing data show in figure 3 starting from 10 neurons to 100 neurons, table 1 shows the results.

Table1 : MLPNN prediction results for one year.

\begin{tabular}{|c|c|c|c|}
\hline \# of Neurons & MSE $_{\text {Train }}$ & MSE $_{\text {Test }}$ & \# of Iteration \\
\hline 10 & $1.87 \mathrm{E}-02$ & $1.93 \mathrm{E}-02$ & 60 \\
\hline 20 & $2.05 \mathrm{E}-02$ & $2.66 \mathrm{E}-02$ & 7 \\
\hline 30 & $9.93 \mathrm{E}-03$ & $1.96 \mathrm{E}-02$ & 33 \\
\hline 40 & $1.11 \mathrm{E}-02$ & $9.97 \mathrm{E}-03$ & 8 \\
\hline 50 & $5.44 \mathrm{E}-03$ & $1.18 \mathrm{E}-02$ & 13 \\
\hline 60 & $5.54 \mathrm{E}-03$ & $6.73 \mathrm{E}-03$ & 8 \\
\hline 70 & $2.76 \mathrm{E}-03$ & $1.61 \mathrm{E}-02$ & 11 \\
\hline 80 & $3.28 \mathrm{E}-03$ & $4.91 \mathrm{E}-03$ & 14 \\
\hline 90 & $1.78 \mathrm{E}-03$ & $1.08 \mathrm{E}-02$ & 12 \\
\hline 100 & $2.81 \mathrm{E}-03$ & $6.49 \mathrm{E}-03$ & \\
\hline
\end{tabular}


As can be seen from the results obtained in Table 1, the proposed model reduces the error significantly, which means that the MLPNN model is capable of predicting the future electricity consumption. As the table shows the best number of neurons is 90 with lowest MSE equal to $1.78 \mathrm{e}-03$, with a reasonable number of iteration figure3shows the fitting function.

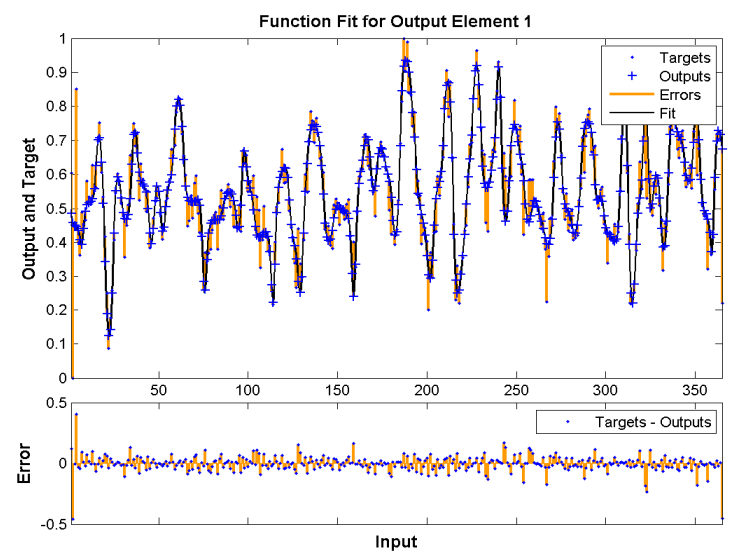

Figure.3. Fitting Result of one Year Prediction.

It is perfectly clear from Figure 3 that as the number of input data increases the model needs a suitable number of neurons. The prediction is able to take more complicated forms and better adapt to changes in the electricity consumption during 12 months. However, it is also noted that so in some input data the prediction is not very efficient, this because in these input data values, the electricity consumption changes fast. Therefore, it will be necessary to stop the prediction when we use a suitable number of neurons so that the prediction obtained is as accurate as possible.

\subsection{MONTHLY FORECASTING EXPERIMENT}

For month forecasting, we use data average for last four January and July month from (20112014), the most electrical consumption figure 4 shows the consumption in two months.
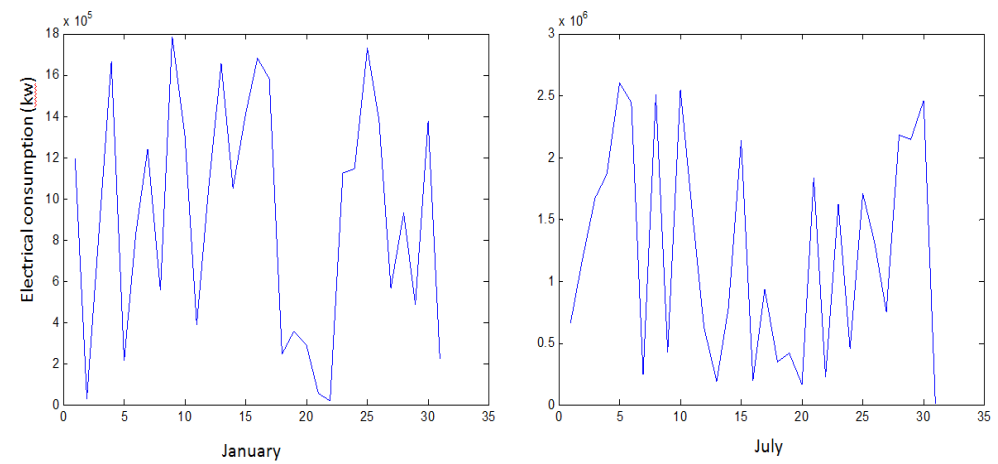

Figure.4. Electrical Consumption for January and July Months 
International Journal of Artificial Intelligence and Applications (IJAIA), Vol.8, No.2, March 2017

Table.2. Neural network result for month forecasting

\begin{tabular}{|c|c|c|c|c|c|c|}
\hline & \multicolumn{3}{|c|}{ January } & \multicolumn{3}{|c|}{ July } \\
\hline $\begin{array}{l}\text { \# of } \\
\text { Neurons }\end{array}$ & $\mathbf{M S E}_{\text {Train }}$ & MSE $_{\text {Test }}$ & $\begin{array}{c}\text { \# of } \\
\text { Iteration }\end{array}$ & $\mathbf{M S E}_{\text {Train }}$ & MSE $_{\text {Test }}$ & $\begin{array}{c}\text { \# of } \\
\text { Iteration }\end{array}$ \\
\hline 2 & $4.55 \mathrm{E}-02$ & $8.84 \mathrm{E}-02$ & 7 & $9.96 \mathrm{E}-02$ & $1.39 \mathrm{E}-01$ & 6 \\
\hline 4 & 4.63E-02 & $2.07 \mathrm{E}-01$ & 7 & $3.48 \mathrm{E}-03$ & $1.39 \mathrm{E}-03$ & 43 \\
\hline 6 & $2.75 \mathrm{E}-02$ & $8.06 \mathrm{E}-03$ & 8 & $3.64 \mathrm{E}-02$ & $2.23 \mathrm{E}-02$ & 7 \\
\hline 8 & $4.74 \mathrm{E}-02$ & $2.27 \mathrm{E}-02$ & 7 & $2.25 \mathrm{E}-02$ & $1.16 \mathrm{E}-02$ & 7 \\
\hline 10 & $2.90 \mathrm{E}-02$ & $7.10 \mathrm{E}-03$ & 7 & $4.68 \mathrm{E}-03$ & $1.14 \mathrm{E}-02$ & 10 \\
\hline 12 & $3.08 \mathrm{E}-02$ & $5.86 \mathrm{E}-03$ & 8 & $3.74 \mathrm{E}-03$ & $2.34 \mathrm{E}-01$ & 7 \\
\hline 14 & $1.53 \mathrm{E}-02$ & $9.93 \mathrm{E}-02$ & 8 & $2.33 \mathrm{E}-03$ & $7.58 \mathrm{E}-02$ & 8 \\
\hline 16 & $9.62 \mathrm{E}-04$ & $1.03 \mathrm{E}-01$ & 9 & $8.44 \mathrm{E}-04$ & $5.31 \mathrm{E}-02$ & 10 \\
\hline 18 & $1.58 \mathrm{E}-02$ & $1.31 \mathrm{E}-02$ & 7 & $1.81 \mathrm{E}-02$ & $8.90 \mathrm{E}-02$ & 7 \\
\hline 20 & $3.72 \mathrm{E}-03$ & $1.85 \mathrm{E}-02$ & 10 & $3.87 \mathrm{E}-03$ & $3.58 \mathrm{E}-02$ & 5 \\
\hline 22 & $3.41 \mathrm{E}-03$ & $8.47 \mathrm{E}-02$ & 6 & $4.81 \mathrm{E}-03$ & $1.21 \mathrm{E}-01$ & 7 \\
\hline 24 & $3.20 \mathrm{E}-14$ & $2.07 \mathrm{E}-01$ & 4 & $1.18 \mathrm{E}-15$ & $7.68 \mathrm{E}-02$ & 5 \\
\hline
\end{tabular}

The result of applying the data after pre-processing in network model starting from 2 neurons to 24 neurons show in the following table. As we see in the table the best number of neurons is 24 in both months with lowest MSE equal to $3.20 \mathrm{e}^{-14}, 1.18 \mathrm{e}^{-15}$ respectively, figure 5shows the fitting function for January month and figure 6shows the fitting function for July month.

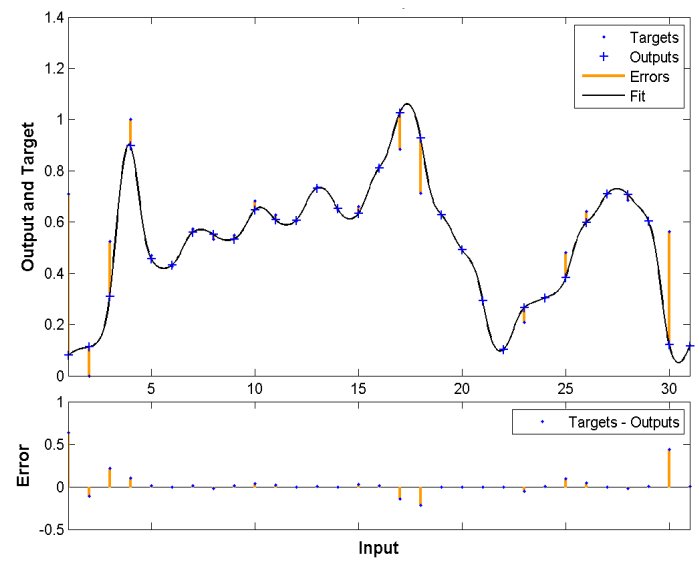

Figure.5.Fitting Result of January.

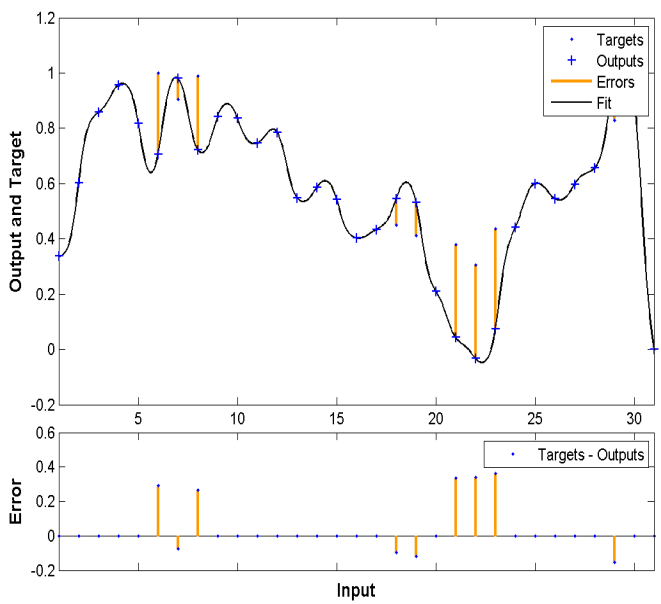

Figure.6.Fitting Result of July 
International Journal of Artificial Intelligence and Applications (IJAIA), Vol.8, No.2, March 2017

From Figures 4 and 5, which presents the prediction process of January and July, these two months proposed to be the maximum 2 months of electricity consumption in Palestine? It's clear that the proposed model predict well this maximum electricity consumption during the year. It is observed that the electricity consumption prediction is well in both months. The difference between the two predictions cannot be observed by the graphs and can only measure of the errors obtained.

\section{CONCLuSiON}

In this paper, we try to forecast the electrical power consumption according to effective factors by using the artificial neural network, also trying to find out the pattern of electrical power consumption with the dataset which is prepared by real data collected from Nablus city at the north of Palestine. The model which used in this paper can be helpful to manage the distribution of electrical power and manage usage of it. The result suggests that MLPNNBP model can be used as an important tool to perform a good prediction with least mean square error as short load forecasting model. The predictions, as it has been shown, are very beneficial using neural networks with the condition to apply a pre-processing to the real collected data. The proposed model has been able to predict the electricity consumption with an accuracy of more than $95 \%$, which appears in the small mean square error. Finally, the main objective of this project was to predict the electricity consumption in Palestine using a neural network model that provides accurate predictions on the electricity, which helps the electrical companies and responsible authorities to plan for the future of the electricity in Palestine. With regard to future work, there are several aspects to be analyzed. The first is the incorporation of MLPNN with optimization algorithms like genetic algorithms. Filtering data algorithms will be used to solve a problem that many data in the electricity consumption time series were points atypical due to problems of measurement of demand.

\section{REFERENCES}

[1] Kandananond, Karin. "Forecasting electricity demand in Thailand with an artificial neural network approach." Energies 4.8 (2011): 1246-1257.

[2] Kargar, mohammadjavad, and D. K. Charsoghi. "Predicting Annual Electricity Consumption In Iran Using Artificial Neural Networks (Narx)." Indian J. Sci. Res 5.1 (2014): 231-242.

[3] Ibrik, Imad H., and Marwan M. Mahmoud. "Energy efficiency improvement procedures and audit results of electrical, thermal and solar applications in Palestine." Energy Policy 33.5 (2005): 651-658.

[4] Abualkhair, Ayman. "Electricity sector in the Palestinian territories: Which priorities for development and peace?." Energy policy 35.4 (2007): 2209-2230.

[5] Gaillard, Pierre, and YannigGoude. "Forecasting electricity consumption by aggregating experts; how to design a good set of experts." Modeling and Stochastic Learning for Forecasting in High Dimensions. Springer International Publishing, 2015. 95-115.

[6] Wang, Long, Zijun Zhang, and Jieqiu Chen. "Short-Term Electricity Price Forecasting with Stacked DenoisingAutoencoders." IEEE Transactions on Power Systems (2016).

[7] Kaytez, Fazil, et al. "Forecasting electricity consumption: a comparison of regression analysis, neural networks, and least squares support vector machines." International Journal of Electrical Power \& Energy Systems 67 (2015): 431-438.

[8] Nahari, Ali, Habib Rostami, and Rahman Dashti. "Electrical Load Forecasting in Power Distribution Network by Using Artificial Neural Network." International Journal of Electronics Communication and Computer Engineering 4.6 (2013): 1737-1743.

[9] Samhouri, M., A. Al-Ghandoor, and R. Fouad. "Electricity Consumption in the Industrial Sector of Jordan: Application of Multivariate Linear Regression and Adaptive Neuro-Fuzzy Techniques." POWER CONTROL AND OPTIMIZATION: Proceedings of the Second Global Conference on Power Control and Optimization. Vol. 1159. No. 1. AIP Publishing, 2009.

[10] Simon S. Haykin (1998), Neural Networks-A Comprehensive Foundation, 2nd edn. Prentice Hall New Jersey, USA, pp286-289. 
International Journal of Artificial Intelligence and Applications (IJAIA), Vol.8, No.2, March 2017

[11] K.Hornik, M. Stinchcombe, and H.White, "Multilayer feed forward networks are universal approximators," Neural Networks, vol. 2, no. 5, pp. 359-366, 1989.

[12] Wang, Wen-Chuan, et al. "A comparison of performance of several artificial intelligence methods for forecasting monthly discharge time series." Journal of hydrology 374.3 (2009): 294-306

[13] H. Gavin, "The Levenberg-Marquardt method for nonlinear least squares curve-fitting problems, Department of Civil and Environmental Engineering Duke University. Jan. 2009

[14] Qasrawi, I, Awad. M, "Prediction of the Power Output of Solar Cells Using Neural Networks: Solar Cells Energy Sector in Palestine" International Journal of Computer Science and Security (IJCSS), Volume (9) : Issue (6) , 280-292, 2015.

[15] Ekonomou, L. "Greek long-term energy consumption prediction using artificial neural networks." Energy 35.2 (2010): 512-517.

[16] Yuan, Chaoqing, Sifeng Liu, and Zhigeng Fang. "Comparison of China's primary energy consumption forecasting by using ARIMA (the autoregressive integrated moving average) model and GM $(1,1)$ model." Energy 100 (2016): 384-390.

[17] Chae, Young Tae, et al. "Artificial neural network model for forecasting sub-hourly electricity usage in commercial buildings." Energy and Buildings 111 (2016): 184-194.

[18] Li, Kangji, et al. "Building's electricity consumption prediction using optimized artificial neural networks and principal component analysis." Energy and Buildings 108 (2015): 106-113.

[19] Karanfil, Fatih, and Yuanjing Li. "Electricity consumption and economic growth: exploring panelspecific differences." Energy Policy 82 (2015): 264-277.

[20] Biswas, MA Rafe, Melvin D. Robinson, and Nelson Fumo. "Prediction of residential building energy consumption: A neural network approach." Energy117 (2016): 84-92.

[21] M. T. Hagan, H. B. Demuth, and M. Beale, Neural Network Design, PWS Publishing Company, Boston, Mass, USA, 1995.

[22] Box, George EP, et al. Time series analysis: forecasting and control. John Wiley \& Sons, 2015. 